\title{
Regulation of functional steroid receptors and ligand-induced responses in telomerase- immortalized human endometrial epithelial cells
}

\author{
S Hombach-KIonisch ${ }^{1}$, A Kehlen ${ }^{2}$, P A Fowler ${ }^{3}$, B Huppertz ${ }^{4}$, J F Jugert ${ }^{5}$, G Bischoff ${ }^{6}$, \\ E Schlüter', J Buchmann ${ }^{7}$ and T Klonisch' \\ ${ }^{1}$ Department of Human Anatomy and Cell Science, University of Manitoba, Winnipeg, Manitoba, Canada \\ ${ }^{2}$ Department of Immunology, Martin Luther University, Halle-Wittenberg, Germany \\ ${ }^{3}$ Department of Obstetrics and Gynecology, University of Aberdeen, Aberdeen, UK \\ ${ }^{4}$ Department of Anatomy, RWTH Aachen, Aachen, Germany \\ ${ }^{5}$ Department of Dermatology, RWTH Aachen, Aachen, Germany \\ ${ }^{6}$ Department of Analytical and Environmental Chemistry, Martin Luther University, Halle-Wittenberg, Germany \\ ${ }^{7}$ Department of Pathology, Martin Luther University, Halle-Wittenberg, Germany
}

Correspondence should be addressed to T Klonisch

Email

Thomas.Klonisch@med.

umanitoba.ca

The authors wish to alert readers of the above paper, which appeared in volume 34 , part 2, pages 517-534, to the following:

Published DNA profiling data identified hTERT-EEC cells as misidentified MCF7 breast cancer cells (Korch et al. 2012; Gynecologic Oncology 127 241-248; doi:10.1016/j.ygyno.2012.06.017). We cannot exclude that the cells used in this paper are a MCF7 contamination and therefore advise caution in the interpretation of the data reported. 